\title{
Perspective critique sur l'enseignement de la littératie disciplinaire en contexte d'inclusion et incidences sur la planification de l'enseignement
}

NANCY GRANGER

Université de Sherbrooke

ANDRÉ C. MOREAU

Université du Québec en Outaouais

\section{Résumé}

Cet article porte sur la littératie disciplinaire et propose un modèle intégrateur des interventions pédagogiques facilitant la compréhension et l'appropriation de contenu chez les élèves (Hillman, 2014). Faisant suite à une synthèse des connaissances sur la littératie disciplinaire réalisée par Granger et Moreau (2018), deux recensions permettront de nuancer les apports d'une part de la littératie disciplinaire (Hillman, 2014), et d'autre part de la littératie générale (Flaggella-Luby, Sampson Graner, Deshler et Valentino Drew, 2012) dans la planification de l'enseignement au bénéfice d'élèves en difficulté d'apprentissage. Tout au long de cet article, des exemples sont apportés et quelques recommandations sont formulées dans le but de guider les enseignants qui souhaitent rehausser les compétences en littératie chez leurs élèves de la fin du primaire ou du secondaire.

Mots-clés

Littératie disciplinaire, littératie générale, interventions, enseignants, élèves en difficulté d'apprentissage.

\section{Abstract}

This article focuses on disciplinary literacy and proposes an integrative model of instructional interventions that facilitate students' understanding and appropriation of content (Hillman, 2014). Following a synthesis of knowledge on Granger and Moreau's (2018) disciplinary literacy, two reviews will qualify the contributions of both disciplinary literacy (Hillman, 2014) and general literacy. (Flagella-Luby Graner, Deshler and Valentino Drew, 2012) in educational planning for the benefit of students with learning difficulties. Throughout this article, examples are provided and some recommendations are made to guide teachers who wish to enhance literacy skills in their students at the end of primary or secondary school.

Keywords

Disciplinary literacy, content area literacy, interventions, teacher, students with learning disabilities

Introduction

La présente recension intégrative d'écrits scientifiques s'inscrit en continuité avec un premier article qui portait sur Les recensions antérieures de recherches sur les enseignements favorisant les compétences en littératie de la fin du primaire et du 
secondaire (Granger et Moreau, soumis). Outre les interventions ${ }^{1}$ pédagogiques documentées dans cette dernière, il apparaît nécessaire de s'intéresser au comment faire, c'est-à-dire aux méthodes ${ }^{2}$ utilisées en enseignement de la littératie (Messier, 2014). Ces méthodes constituent une excellente voie pour soutenir l'apprentissage en contextes scolaires inclusifs du primaire et du secondaire.

Récemment, le Bureau international d'éducation (BIE) a produit un rapport sur les principaux enjeux actuels en matière de curriculum et d'apprentissage. L'auteur rappelle que l'efficacité de l'apprentissage passe par la mobilisation cognitive des élèves ; il s'agit de rendre ces derniers les plus actifs possible, et ce, le plus souvent possible à travers des situations complexes en salle de classe (Roegiers, 2016, p. 11). Des compétences transversales comme traiter ou communiquer de l'information peuvent être mises à profit pour construire du sens autour des savoirs et des savoir-faire disciplinaires. Pour y arriver, les élèves devront mobiliser des ressources telles que des processus et des stratégies d'apprentissage ou encore des concepts qui leur auront été enseignés (Ministère de l'Éducation du Québec [MEQ], 2003, p. 4).

Bien que les compétences soient généralement perçues comme étant la responsabilité des élèves, il demeure que l'enseignant a un rôle prépondérant dans la planification de situations qui leur fourniront de multiples occasions d'apprendre de nouvelles connaissances et compétences afin qu'ils les intègrent à celles qu'ils possèdent déjà. Les approches basées sur la résolution de problèmes sont notamment connues pour contribuer à l'optimisation du potentiel des apprenants. Pourtant, selon Jonnaert (2010), la tendance actuelle consisterait à laisser à l'enseignant la liberté de choisir les modalités pédagogiques pour mener les apprentissages, quelle que soit l'optique dans laquelle les curricula officiels sont rédigés. Or, la présente recension montre que les pratiques d'enseignement ont beaucoup à voir avec la qualité des apprentissages des élèves et ne peuvent être laissées qu'au libre choix du personnel enseignant. Elles doivent s'inscrire dans une vision plus large de l'apprentissage qui implique nécessairement les concepteurs de programmes, les formateurs d'enseignants, les directions d'établissement et tout le personnel de l'école (Granger et Moreau, soumis).

Prenant appui de cette première recension, les objectifs de cet article consistent à 1) rendre explicites les apports de la littératie disciplinaire comme complément à la littératie générale; et 2) clarifier l'incidence de ces savoirs sur la planification de l'enseignement.

Pour traiter de ces enjeux, deux recensions d'écrits scientifiques sont analysées selon une perspective critique de l'enseignement de la littératie disciplinaire en contexte d'inclusion. L'article de Hillman (2014) porte sur la littératie disciplinaire et propose un

\footnotetext{
${ }^{1}$ Le terme intervention utilisé dans ce texte s'appuie sur la définition de Lenoir (2009) et réfère à l'agir de l'enseignant dans ses rapports aux élèves. Sur le plan empirique, opérationnel et pragmatique, l'intervention pédagogique renvoie à l'action de l'enseignant en interaction vers un apprenant ou un groupe d'élèves et vers les savoirs. L'enseignant met en place des dispositifs et processus médiateurs en tenant compte des finalités diverses des acteurs (parents, directions d'établissement, personnel scolaire) et des composantes qui fondent cette interaction apprenant-savoir-enseignant; cette définition impose un rapport multiple, objectif et subjectif, sur l'acte d'enseignement et l'acte d'apprendre.

${ }^{2}$ Comme le souligne Messier (2014), l'usage du terme générique « méthode » renvoie à différents concepts, dont approche, pratique, technique, modalité, dispositif, formule, stratégie, intervention qui visent à fournir des ressources didactiques et pédagogique. Cette auteure précise dans sa thèse les nuances entre ces termes. Dans le présent texte, le terme méthode désigne « une organisation codifiée de techniques et de moyens mis en œuvre pour atteindre un ou des objectifs » (Messier, 2014, p. 129).
} 
modèle intégrateur des interventions pédagogiques facilitant la compréhension et l'appropriation de contenu chez les élèves (Hillman, 2014). L'article de Flaggella-Luby, Sampson Graner, Deshler et Valentino Drew (2012) vise à démontrer les tenants et aboutissants des interventions en littératie générale et les comparer avec ceux en littératie disciplinaire dans le cas d'élèves en difficulté d'apprentissage. Cette analyse nous permet de préciser les incidences sur la planification de l'enseignement et de faire quelques recommandations aux enseignants.

\section{La démarche de recension des écrits scientifiques}

Afin de documenter le sens de la littératie disciplinaire et les interventions qui favorisent son développement, nous avons réalisé une recension intégrative des écrits (Fortin et Gagnon, 2016 ; Jackson, 1980) en interrogeant l'outil EBSCO qui comprend plusieurs banques de données en éducation, dont Academic Search Complete, Education Source et ERIC. Différents mots-clés ont permis la recension de recherches parues dans des revues universitaires avec comité de lecture et impliquant des élèves de la fin du primaire, du début secondaire ou du secondaire (grade 4 to 8; middle school, secondary level, $K-12)$. Notre recherche est basée sur les mots-clés suivants : « disciplinary literacy et review, overview or meta-analysis ». Au total, soixante-six articles correspondent à ces termes et couvrent la période après 2005 jusqu'à 2017. Afin de circonscrire notre recherche aux élèves de la fin du primaire et du début du secondaire, les mots-clés « middle school or secondary school» ont été ajoutés, ne laissant que huit textes à consulter. Après vérification, un seul texte correspond à notre intérêt de recherche, soit de répertorier dans les revues universitaires révisées par les pairs d'articles sur les recherches en littératie disciplinaire. Nous utiliserons donc le texte d'Hillman (2014) comme point de départ pour définir ce qu'est la littératie disciplinaire et pour comprendre quelles sont les compétences associées au développement de ce type de littératie chez les élèves de la fin du primaire et du secondaire. Nous ferons ensuite le lien avec l'article de Flaggella-Luby et al. (2012) qui propose de faire cohabiter en enseignement la littératie générale avec la littératie disciplinaire. Le point de vue de ces auteurs apporte un éclairage pertinent quant aux interventions à valoriser auprès les élèves en difficulté d'apprentissage. Nous réinvestirons les propos des différents auteurs rassemblés dans ces recensions antérieures pour répondre à deux questions laissées en suspens dans notre premier article c'est-à-dire : a) Quels peuvent être les apports de la littératie disciplinaire comme complément à la littératie générale ? et b) Quelle est l'incidence de ces savoirs sur les compétences en enseignement ?

\section{Traiter de littératie disciplinaire ne peut se faire sans traiter de la littératie générale}

L'Organisation de Coopération et de Développement Économiques (OCDE, 2000, p. 12) définit la littératie comme «l'aptitude à comprendre et à utiliser l'information écrite dans la vie courante, à la maison, au travail et dans la collectivité en vue d'atteindre des buts personnels et d'étendre ses connaissances et ses capacités ». Les compétences en littératie, lecture et écriture, servent entre autres à mieux communiquer et à développer l'esprit critique des personnes qui vivent en société (Thévenaz-Christen, 2011). En francophonie, les recherches contribuent à enrichir ce concept de littératie. Elles mettent en valeur une vision compréhensive de l'être humain, de sa capacité à interagir dans les milieux et au sein de la communauté. Ces recherches promeuvent l'utilisation de moyens et de supports variés de langages pour optimiser le potentiel des personnes dans une visée 
de participation sociale et d'une contribution citoyenne (Moreau, Hébert, Lépine et Ruel, 2013). Ainsi, au terme de leur recension des écrits en francophonie, ces chercheurs ont défini la littératie comme la capacité de lire, d'écrire et de communiquer efficacement, sur différents supports afin de se développer et de fonctionner dans le quotidien en réalisant ses buts et en exploitant son potentiel.

Loin d'être fixe, cette définition est en évolution au regard des nouvelles connaissances; à cet égard, le Réseau québécois sur la littératie ${ }^{3}$ (Lacelle, Lafontaine, Moreau et Laroui, 2016) apporte un éclairage plus large en proposant que le terme de littératie, en francophonie, réfère à la capacité d'une personne, d'un milieu et d'une communauté à comprendre et à communiquer de l'information par le langage sur différents supports pour participer activement à la société dans différents contextes. Ainsi, comprendre et communiquer de l'information supposent la mobilisation de ressources (connaissances et compétences) cognitives, linguistiques, graphiques, affectives, expérientielles, culturelles et techniques pour décoder, lire, écouter, se représenter, réfléchir, interpréter, sélectionner, analyser, évaluer, s'exprimer, interagir, créer, produire, diffuser, critiquer et résoudre des problématiques au quotidien (Lacelle et al., 2016).

Dans le parcours scolaire, l'acquisition de compétences en littératie renvoie à un continuum d'acquis qui va en se complexifiant du primaire au secondaire. Shanahan et Shanahan (2008) précisent trois niveaux de littératie selon l'analogie d'une pyramide de compétences : a) le niveau de base de compétences en littératie (lire et écrire) implique des habiletés telles que le décodage et la connaissance des mots à haute fréquence qui sont sollicitées pratiquement dans toutes les tâches de lecture et d'écriture; b) le niveau intermédiaire de littératie comprend des compétences communes à de nombreuses tâches comme trouver le sens des mots commun, développer la fluidité en lecture et en écriture ou encore, chercher, traiter, analyser de l'information en référant à ses connaissances antérieures ou en mettant à profit des stratégies d'apprentissage utiles en lecture et en écriture telles que survoler, surligner ou annoter le texte lu; et c) le niveau supérieur propre à la littératie disciplinaire est fondé sur les compétences en littératie générale transposées dans des situations complexes (Roegiers, 2010 ; Tardif, 2006), ou en contexte authentique (Wiggins, 1993) au sein desquels les élèves mobilisent leurs savoirs, leurs savoir-agir et leurs savoir-être dans des tâches d'intégration (Roegiers, 2016). Organisées selon des modalités variées, interactives et adéquates, les tâches d'intégration permettent, au terme d'un enseignement explicite des compétences en littératie de base et intermédiaire, d'évaluer la capacité des élèves à utiliser ses compétences développées en situation autonome et spécifique à une discipline.

En contexte québécois, les compétences énumérées dans le Programme de formation de l'école québécoise, secondaire, premier cycle (MEQ, 2003) suggèrent que les élèves doivent avoir développé des compétences en littératie pour répondre aux exigences ministérielles. Prenons l'exemple de trois compétences en science et en technologie au secondaire illustrées dans le tableau 1 ci-dessous. Pour chacune d'entre elles, les élèves doivent pouvoir lire, extraire de l'information, utiliser ces dernières pour formuler des réponses à des questions, ou pour approfondir leur compréhension des contenus. Les compétences en littératie générale leur permettent d'effectuer les tâches de base qui leur sont demandées. Toutefois, en situation complexe, ceux-ci sont confrontés à des demandes spécifiques par exemple, en sciences, justifier pourquoi choisir tel matériau

\footnotetext{
${ }^{3}$ Réseau de plusieurs chercheurs québécois sous la direction de Lacelle et al. (2016).
} 
pour produire une nouvelle prothèse à un nageur olympique afin d'améliorer ses performances. Pour faire une proposition plausible et pertinente, les élèves doivent se référer au vocabulaire spécifique de la discipline enseignée et recourir aux connaissances antérieures sur les termes impliqués. Notamment, en science et technologie, les élèves peuvent comparer des indices d'étanchéité des matériaux, de flottabilité, de malléabilité et de résilience. Pour les justifier, ils doivent lire des textes sur le sujet, comprendre des tableaux, en produire à leur tour, le tout en utilisant la démarche scientifique. Le tableau 1 illustre la concordance entre les compétences du MEQ (2003) et les discours en littératie générale et en littératie disciplinaire selon Hillman.

Tableau 1

Regard croisé entre compétences du MEQ (2003) et le discours en littératie générale et disciplinaire inspiré de Hillman (2014)

\begin{tabular}{|c|c|c|}
\hline $\begin{array}{l}\text { Compétences en science et } \\
\text { technologie (MEQ, 2003) }\end{array}$ & $\begin{array}{l}\text { Discours en littératie } \\
\text { générale (Hillman, 2014) }\end{array}$ & $\begin{array}{l}\text { Discours en littératie } \\
\text { disciplinaire (Hillman, } \\
\text { 2014) }\end{array}$ \\
\hline $\begin{array}{l}\text { Mettre à profit ses } \\
\text { connaissances scientifiques } \\
\text { et technologiques }\end{array}$ & $\begin{array}{l}\text { - Mobiliser ses compétences } \\
\text { en littératie : } \\
\text { communiquer, lire et écrire } \\
\text { avec fluidité et utiliser ses } \\
\text { connaissances pour } \\
\text { résoudre des problèmes ou } \\
\text { pour réaliser des tâches }\end{array}$ & $\begin{array}{l}\text { - Mobiliser ses } \\
\text { compétences spécialisées } \\
\text { dans les domaines } \\
\text { disciplinaires et les } \\
\text { sujets spécifiques tels } \\
\text { qu'en science et } \\
\text { technologie.... }\end{array}$ \\
\hline $\begin{array}{l}\text { Chercher des réponses ou } \\
\text { des solutions à des } \\
\text { problèmes d'ordre } \\
\text { scientifique et } \\
\text { technologique }\end{array}$ & $\begin{array}{l}\text { disciplinaires } \\
\text { - Mettre à profit ses } \\
\text { compétences et } \\
\text { connaissances communes } \\
\text { en communication orale et } \\
\text { écrite dans diverses tâches } \\
\text { disciplinaires }\end{array}$ & $\begin{array}{l}\text { - Résoudre des problèmes } \\
\text { à la manière d'experts } \\
\text { - Écrire, discuter et étayer } \\
\text { sa pensée } \\
\text { - Passer de la lecture du } \\
\text { texte à la représentation } \\
\text { visuelle pour illustrer sa }\end{array}$ \\
\hline $\begin{array}{l}\text { Communiquer à l'aide des } \\
\text { langages utilisés en science } \\
\text { et technologie }\end{array}$ & & $\begin{array}{l}\text { compréhension (littératie } \\
\text { visuelle et numérique) } \\
\text { - S'approprier différents } \\
\text { genres de communication } \\
\text { - S'approprier les normes } \\
\text { d'édition }\end{array}$ \\
\hline
\end{tabular}

Comme le soulignent Perle, Grigg et Donahue (2005), ce n'est pas parce les élèves ont utilisés des stratégies d'apprentissage générales pour traiter des textes au primaire qu'ils ont les compétences nécessaires pour aborder des textes spécialisés en littérature, en science et technologie ou en histoire au secondaire. Un enseignement explicite des conventions, du vocabulaire spécialisé, des méthodes pour synthétiser et pour développer un raisonnement propre à la discipline s'avère nécessaire pour réaliser des tâches de plus en plus complexes dans les disciplines scolaires (Shanahan et Shanahan, 2008, p. 4). 
Dans la présente démarche documentaire de recherche sur l'enseignement de compétences en littératie disciplinaire, nous avons interrogé les écrits scientifiques afin d'analyser ces textes sur les interventions en enseignement de la littératie disciplinaire.

\section{L'apport de recherches sur les interventions qui favorisent les compétences en littératie disciplinaire}

L'analyse des textes recensés mène à documenter deux types d'écrits : d'une part, les recensions antérieures et les recherches sur la littératie disciplinaire et les interventions qui favorisent l'appropriation de ces compétences au primaire et au secondaire ; et d'autre part, les auteurs qui développent un argumentaire critique du concept de la littératie générale. La section qui suit porte sur la littératie disciplinaire et les interventions en enseignement primaire et secondaire qui les favorisent; la dernière section présente un point de vue critique de la littératie générale et des interventions à privilégier en contexte d'enseignement disciplinaire. Une synthèse des éléments recensés termine cette section.

\section{La littératie disciplinaire et les interventions qui la favorisent}

Selon les tenants de la littératie disciplinaire, il ne suffit pas d'enseigner des stratégies d'apprentissage générales en lecture pour que les élèves apprennent comment comprendre les textes spécifiques aux disciplines. Pour rendre compte des avancées de la recherche dans le champ de la littératie disciplinaire, Hillman (2014) a réalisé une revue de littérature dans son domaine, soit en mathématiques, dans laquelle il dresse un inventaire d'articles révisés par les pairs, de livres en éducation et des rapports concernant l'enseignement des mathématiques au secondaire depuis les années 2000. Elle a interrogé les écrits recensés sous trois angles soit a) Comment la littératie disciplinaire amène-t-elle les élèves à apprendre ?; b) Comment le Common Core State Standard (CCSS) conçoit l'apport de la littératie disciplinaire en mathématiques ?; et c) Comment le programme de formation intègre l'enseignement de la littératie disciplinaire en classe de mathématiques ?

En réponse à sa première question sur l'apport de la littératie sur l'apprentissage, Hillman relève que les situations d'apprentissage proposées aux élèves dans une perspective de développement de compétence en littératie disciplinaire placent ces derniers dans des contextes authentiques afin qu'ils s'approprient une forme de discours disciplinaire par la lecture, la réflexion, la discussion et l'écriture. Ces situations d'apprentissage ont pour but de rendre compte de la compréhension de l'élève. Pour illustrer comment ces apprentissages se réalisent, l'auteure fait un parallèle entre le langage utilisé en mathématiques, les interventions en mathématiques du CCSS et les interventions valorisées par les programmes de formation. Par exemple, la lecture du CCSS suggère que lorsque les élèves abordent une situation problème en mathématiques, ils doivent pouvoir créer du sens avec les données qui leur sont proposées en persévérant dans leur démarche de résolution de problème. Du côté des programmes en mathématiques, les auteurs suggèrent que la résolution de problème se réalise par l'utilisation d'approches variées, dont la visée est de permettre aux élèves de développer la capacité à s'autoréguler ${ }^{4}$. En outre, le discours associé à la littératie disciplinaire encourage le recours à des séquences d'enseignement-apprentissage authentiques dont le contexte et les tâches sont réels et

${ }^{4}$ S'autoréguler renvoie à l'ensemble des actions ou processus cognitifs et métacognitifs menant l'apprenant à maintenir un équilibre ou à s'ajuster pour atteindre ses objectifs ou buts personnels (Zimmerman et Schunk, 2001). 
complexes (Passmore, 2007). Les enjeux de ce contexte et ces tâches ressemblent à ceux que vivent des experts de la discipline afin que les élèves acquièrent des compétences et connaissances propres à cette discipline, en l'occurrence ici, en mathématiques. En présentant des situations authentiques, l'enseignant permet aux élèves de nommer, d'expliquer, de justifier qu'ils intégreront les savoirs et savoir-faire disciplinaires et qu'ils développeront du sens (Gee, 2012; Shanahan et Shanahan, 2008). La démarche itérative de ces situations incite les élèves à chercher de l'information et les habilite à étayer leur pensée (Bostic et Matney, 2013).

Pour répondre à sa deuxième question sur le comment enseigner, Hillman (2014) propose des modalités d'enseignement et d'apprentissage qui tendent à soutenir le développement d'opérations mentales et de compétences chez les élèves dans une perspective disciplinaire. La représentation visuelle, par des supports tels que les tableaux ou les schémas, engagerait les élèves dans le traitement et dans l'organisation de l'information (Sfrad, 2007). Ces supports visuels sont aussi connus pour soutenir les discussions métacognitives autour du sujet traité. Ces aides visuelles encouragent la justification relative à une résolution de problème ainsi que l'explicitation de liens entre les savoirs, savoir-faire et savoir-être disciplinaires (Wilkerson-Jerde et Wilensky, 2011). Les activités d'interaction entre les élèves sous forme de dialogue et de négociation permettent l'intégration d'un vocabulaire spécialisé et commun, ainsi que l'appropriation de concepts et de contenus disciplinaires qui sont au cœur de la compréhension.

En réponse à sa troisième question sur les interventions pédagogiques préconisées au sein du programme de formation, Hillman (2014) rapporte que les enseignants qui prennent l'habitude de créer ce type de contextes d'enseignement et d'apprentissage aident les élèves à développer leur sens critique en fonction du raisonnement propre à la discipline. Stimuler le questionnement réciproque des élèves et les encourager à prendre du recul face à ce qui leur est exposé permettrait de mieux comprendre le contenu et de se l'approprier (Gresalfi, Martin, Hand et Greeno, 2009; Zorica, Cindric et Destovic, 2012). En résumé, selon la recension effectuée par Hillman (2014), c'est en se familiarisant avec le vocabulaire associé à chaque discipline, les valeurs promues et les contextes culturels dans lesquels les différentes disciplines s'inscrivent que les élèves forgent leur compréhension et développent des habiletés langagières propres à un domaine de compétences. Les discours de l'enseignant et des auteurs qui sont présentés en classe représentent alors des éléments fondateurs de la pensée critique des élèves ; ce qui dépasse l'enseignement d'une littératie générale.

Il demeure toutefois que les études recensées par Hillman (2014) ne s'intéressent pas explicitement aux élèves en difficulté d'apprentissage intégrés en classe ordinaire qui peinent à suivre le contenu comme le rapportaient notamment Kaldenberg, Watt et Therrien (2015) (voir la première recension des écrits dans Granger et Moreau, soumis). À cet égard, Flaggella-Luby et al. (2012) formulent une mise en garde qui va à l'encontre de présenter la littératie disciplinaire en opposition avec l'enseignement de la littératie générale.

Le point de vue critique des tenants de la littératie générale

Comme mentionné ci-dessus, la perspective proposée par les auteurs recensés par Hillman (2014) montre l'importance de bien distinguer deux types de littératie (Shanahan et Shanahan, 2012) : a) la littératie générale (content area literacy) qui concerne les premiers niveaux de compétences; et b) la littératie disciplinaire qui renvoie aux 
connaissances et compétences spécialisées à une discipline et qui font appel aux processus de haut niveau de la pensée dont la pensée critique.

Par ailleurs, Flaggella-Luby et al. (2012) souhaitent montrer que malgré la tendance à prôner un enseignement des stratégies d'apprentissage en littératie disciplinaire, il convient de continuer à enseigner des stratégies de lecture et d'écriture, littératie générale propre aux premiers niveaux d'habiletés en littératie. Ce faisant, cet enseignement permet aux élèves en difficulté de poursuivre leurs apprentissages, et ce, en considérant leurs acquis. Ces auteurs se basent notamment sur les données statistiques en éducation recensées par le National Center for Education Statistics (NCES, 2009). L'analyse de ces données mène à soutenir la conclusion que l'enseignement de stratégies générales du lire et d'écrire demeure le talon d'Achille au secondaire. Selon ce rapport, plus de $60 \%$ des élèves présenteraient des lacunes en lecture ; cette situation freine leurs apprentissages dans les différentes matières, dont celles en enseignement disciplinaire, et risque de nuire à leur réussite scolaire. Plusieurs élèves peineraient à reconnaître l'information littérale dans le texte, à expliciter leur raisonnement, à faire des comparaisons, à trouver les idées principales et secondaires dans un texte, ou encore, à rapporter la trame de fond d'une histoire (Lee, Grigg, et Donahue, 2007).

Flaggella-Luby et al. (2012) ont recensé au total, 31 articles issus de 3 métaanalyses portant sur l'enseignement en lecture et 33 articles issus de 3 recensions en enseignement en écriture qui ont été documentés et analysés. Les articles retenus sont tous issus de revues scientifiques révisées par les pairs et ont été publiés entre 1990-2006 ; ces recherches incluent les élèves entre la $4^{\mathrm{e}}$ année et la $12^{\mathrm{e}}$ année scolaire ayant des difficultés en lecture ou en écriture, et font état d'au moins une mesure de performance reconnue en lecture ou en écriture. Grâce à cette recension, les auteurs souhaitaient : a) faire le point quant aux deux courants en enseignement, l'un s'appuyant sur la littératie disciplinaire et l'autre sur la littératie générale à consolider ; b) identifier les caractéristiques des élèves ayant un trouble de l'apprentissage et voir quelles sont les recommandations qui sont susceptibles de leur être utiles au secondaire; c) préciser les stratégies spécifiques en enseignement de la discipline en compréhension de lecture et en écriture lorsque les élèves ayant un trouble de l'apprentissage sont inclus dans l'échantillon; et, enfin, d) mettre en lumière les impacts des pratiques d'intervention et les pratiques d'organisation de services aux élèves.

En ce qui a trait à leur première question qui traite du courant de la littératie disciplinaire, Flagella-Luby et al. (2012) font état du peu de recherches recensées qui rapportent l'utilisation de stratégies disciplinaires en enseignement. Lorsque les études en font mention, il s'agit principalement de textes en enseignement de la langue soit l'anglais (english language art). Ces recherches se centrent sur lidentification de la structure du texte et sur la trame narrative. Parmi les recherches en écriture, une seule utilise une méthode de raisonnement historique permettant aux élèves de produire un écrit. De plus, les stratégies d'enseignement et d'apprentissage disciplinaires ne sont répertoriées qu'à la fin du primaire et sont peu nombreuses.

En réponse à leur deuxième question portant sur les recherches en littératie disciplinaire qui traitent des élèves en difficulté, Flaggella-Luby et al. (2012) constatent qu'elles sont plutôt absentes du corpus de leur recension.

Leur troisième question visait à savoir quelles étaient les stratégies spécifiques à la discipline en compréhension de lecture et en écriture lorsque les élèves ayant un trouble 
d'apprentissage sont inclus dans l'échantillon. Les auteurs précisent que les études recensées sont pour la plupart réalisées dans des classes à effectifs réduits et que ces données s'avèrent peu ou pas comparables aux données d'élèves de classes ordinaires qui peuvent compter jusqu'à trente élèves. Au regard du type d'études réalisées, les auteurs soulignent que les résultats présentés dans les recherches avec groupe contrôle ne sont pas significatifs et ne font que mettre en lumière le besoin de poursuivre les recherches dans ce sens et de mieux documenter l'impact d'un enseignement explicite auprès d'élèves ayant des difficultés.

Leur dernière question s'intéressait aux impacts des pratiques d'intervention et d'organisation des services aux élèves. Flaggella-Luby et al. (2012) mentionnent que les textes proposés et lus par les élèves dans les recherches recensées ne correspondent pas toujours au niveau de compétences de ces derniers. Ainsi, ces auteurs rappellent l'importance pour les enseignants de connaître la capacité des élèves, ou le niveau de littératie des textes proposés dans les situations d'apprentissage, afin de proposer des textes qui correspondent au niveau de lecture des élèves et ainsi de pouvoir mieux les soutenir dans le passage d'un niveau de texte à un autre (Flagella-Luby et Deshler, 2008).

\section{Que retenir de ces recensions antérieures?}

En résumé, la recension de Flaggella-Luby et al. (2012) ne permet pas de conclure que les stratégies d'enseignement de la littératie disciplinaire sont plus efficaces que les stratégies de littératie générale auprès des élèves ayant des difficultés d'apprentissage. Afin de démontrer la pertinence de ce point de vue, les chercheurs dressent un portrait des interventions reconnues pour soutenir les élèves en difficulté dans leurs apprentissages. Ils soulignent entre autres la mémorisation, l'utilisation de mots-clés et la représentation visuelle qui ont démontré largement leur efficacité (Scruggs et Mastropieri, 2000). D'autres conclusions sont mises en lumière dans cette recension. Nous en faisons état dans les paragraphes suivants.

D'abord, en compréhension en lecture, les travaux de Trabasso et Bouchard (2002) suggèrent que les lecteurs qui sont formés à l'utilisation de stratégies cognitives telles que la visualisation, l'autoquestionnement, l'autorégulation, la vérification d'hypothèses pour réguler leur compréhension ou pour résumer obtiennent des gains significatifs en compréhension par rapport aux élèves qui n'ont pas reçu cet enseignement. Les études comparables ayant été recensées montrent clairement que les élèves en difficulté peuvent apprendre et appliquer efficacement un éventail de stratégies spécifiques aux tâches de lecture, ce qui a des effets positifs sur les mesures de compréhension de la lecture et sur leur performance en classe (Edmonds, Vaughn, Wexler, Reutebuch, Cable et Klingler Tackett., 2009; Graham et Perin, 2007).

Dans un autre ordre d'idées, les recherches recensées en enseignement disciplinaire sur la rédaction de texte-écriture (Harris et Graham, 1999; Schumaker et Deshler, 2009) témoignent de l'apport positif de l'enseignement et de l'apprentissage de stratégies générales en littératie (Flaggella-Luby et al., 2012). Les conclusions de ces recherches montrent que les élèves écrivent davantage de textes et qu'ils peuvent produire des écrits dans différents genres comme la production de rapport, la rédaction d'un essai ou d'un texte argumentatif. La production de ces textes est de meilleure qualité. En conclusion, il y a lieu de croire que les élèves apprennent lorsqu'on leur enseigne des stratégies générales issues de la littératie générale propres à l'écriture de textes de différents genres. 
Comme autre constat, Flaggella-Luby et al. (2012) mettent en évidence les travaux de Bulgren, Deshler et Lenz (2007), qui préconisent la mise en œuvre de séquences d'enseignement-apprentissage menant à l'enrichissement des contenus «content enhancement routines » (CER); ces séquences d'enseignement et d'apprentissage sont présentes sur une longue période. Cette stratégie d'enseignement repose sur les quatre principes fondamentaux suivants : a) les enseignants du domaine disciplinaire doivent sélectionner les caractéristiques essentielles du contenu à enseigner et, ensuite, transposer ce contenu pour ainsi rendre possible l'apprentissage au regard de différents types d'élèves; b) l'enseignement planifié doit répondre aux besoins de la diversité des apprenants qui composent le groupe; c) le processus d'enseignement-apprentissage ne doit pas compromettre l'intégrité du contenu pour accommoder la diversité des élèves; et d) les enseignants et les élèves doivent s'engager dans une interaction de coconstruction ${ }^{5}$ qui respecte le rôle de chacun dans le processus d'apprentissage (Bulgren et al., 2007).

En outre, la planification et la mise en œuvre de cette séquence d'enseignementapprentissage et des principes associés mettent en évidence le rôle central de l'enseignant comme acteur principal. Le but de ces séquences est de réduire les obstacles à l'apprentissage au regard des contenus en introduisant des stratégies de mise en œuvre pouvant être reprises dans différents contextes. Les enseignants ont donc comme tâche de sélectionner les stratégies générales appropriées en lecture et en écriture, de planifier leur utilisation lors d'activités proposées en classe, et ce, de façon récurrente tout au long de l'année afin que les élèves se les approprient. Au sein de leur séquence, les enseignants peuvent incorporer des procédés d'étayage cognitif (Graham et Perin, 2007), des organisateurs graphiques (Nesbit et Adesope, 2006) et susciter l'engagement actif des élèves par la discussion ou la coconstruction au sein de la classe. Enfin, l'observation et l'utilisation fréquente et cohérente de ce modèle aideraient les élèves à développer un ensemble de stratégies générales de haut niveau en littératie pour l'apprentissage du contenu disciplinaire (Bulgren, Lenz, Schumaker, Deshler et Marquis, 2002).

En somme, il existe un certain nombre de recherches sur l'enseignement des stratégies générales en littératie, lecture et écriture, visant les élèves dont ceux ayant une difficulté d'apprentissage ; ces recherches apportent des résultats significatifs quant à la réussite des élèves en enseignement disciplinaire. Les recensions de Hillman (2014) et de Flaggella-Luby et al. (2012) font ressortir les apports de la littératie générale et, dans une moindre mesure, de la littératie disciplinaire en apprentissage. Pour aller plus loin dans la recherche et dans l'enseignement, Flaggella-Luby et al. (2012) suggèrent d'examiner comment les deux visées d'enseignement prenant appui sur la littératie générale ou sur la littératie disciplinaire sont nécessaires à un enseignement de qualité adapté à tous les élèves, dont ceux présentant une difficulté d'apprentissage.

\section{Les enjeux de ces nouvelles connaissances sur la planification de l'enseignement}

Comme présenté plus haut, Shanahan et Shanahan (2008) ont détaillé une relation conditionnelle entre l'apprentissage fondamental nécessaire des stratégies générales de niveau intermédiaire et des stratégies plus avancées de littératie disciplinaire. Des

\footnotetext{
${ }^{5}$ En contexte du travail en équipe collaborative, la coconstruction émerge de l'interaction des personnes en action, en réflexion et qui lie facteurs psychologiques, communicationnels, sociaux et interactionnels (dialogiques) pour résoudre des problèmes communs et pour générer des solutions nouvelles ou pour réfléchir sur le sens de leurs actions (Bourassa, Bélair et Chevalier, 2007).
} 
situations d'évaluation ponctuelles et récurrentes seront proposées pour permettre aux élèves de démontrer le niveau de maitrise atteint quant aux différents savoirs présentés en classe (Roegiers, 2016). Cela suppose un travail de planification inhérent au rôle de l'enseignant qui souhaite développer chez ses élèves des compétences en littératie et ainsi les soutenir en cours d'apprentissage. Une illustration type permet d'illustrer les interventions et les moyens d'enseignement susceptibles d'être intégrés dans un enseignement disciplinaire tout en considérant les besoins des élèves en difficulté d'apprentissage en classe ordinaire.

\section{Un exemple en classe en science et technologie}

Le tableau ci-dessous présente les trois compétences du programme de science et de technologie au secondaire. La première compétence intitulée chercher des réponses ou des solutions à des problèmes d'ordre scientifique invite les élèves à développer des méthodes de travail appropriées aux situations d'apprentissage proposées. Il s'agit de s'approprier des méthodologies propres à la science pour rendre compte de sa démarche (rapport de laboratoire, tableau de données, schémas, etc.). La deuxième compétence consiste à mettre à profit ses connaissances scientifiques et technologiques en recourant aux connaissances déclaratives et procédurales relatives aux contenus suggérés (le modèle atomique, l'organisation du tableau périodique, les composés ioniques, les électrolytes, la concentration, le $\mathrm{pH}$, etc.). Enfin, la troisième compétence vise à communiquer à l'aide des langages utilisés en science et technologie. Ce qui consiste à produire ou à transmettre adéquatement des messages à caractère scientifique ou technologique selon des modalités propres à cette discipline comme l'utilisation du vocabulaire spécifique, l'utilisation de procédés de reformulation, ou la justification à l'oral comme à l'écrit. Le tableau 2 illustre, d'une part, comment les élèves ont un rôle à jouer dans leurs apprentissages et, d'autre part, le rôle important de l'enseignant dans la planification et dans l'orchestration des contenus à apprendre et des stratégies d'enseignement pour y parvenir. 
Tableau 2

Regard croisé des compétences attendues en sciences, des types de littératie, des moyens et stratégies d'enseignement

\begin{tabular}{|c|c|c|c|c|c|c|}
\hline 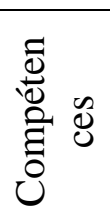 & \multicolumn{2}{|c|}{$\begin{array}{l}\text { 1. Chercher des réponses ou des } \\
\text { solutions à des problèmes } \\
\text { d'ordre scientifique ou } \\
\text { technologique }\end{array}$} & \multicolumn{2}{|c|}{$\begin{array}{l}\text { 2. Mettre à profit ses connaissances } \\
\text { scientifiques et technologiques }\end{array}$} & \multicolumn{2}{|c|}{$\begin{array}{l}\text { 3. Communiquer à l'aide des langages } \\
\text { utilisés en sciences et en technologies }\end{array}$} \\
\hline 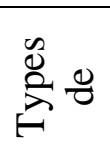 & $\begin{array}{l}\text { Discours de la } \\
\text { littératie } \\
\text { générale }\end{array}$ & $\begin{array}{l}\text { Discours de la } \\
\text { littératie } \\
\text { disciplinaire }\end{array}$ & $\begin{array}{c}\text { Discours de la } \\
\text { littératie générale }\end{array}$ & $\begin{array}{l}\text { Discours de la } \\
\text { littératie } \\
\text { disciplinaire }\end{array}$ & $\begin{array}{l}\text { Discours de la } \\
\text { littératie } \\
\text { générale }\end{array}$ & $\begin{array}{l}\text { Discours de la } \\
\text { littératie disciplinaire }\end{array}$ \\
\hline 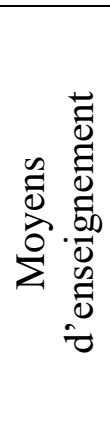 & $\begin{array}{l}\text { - Utiliser des } \\
\text { approches } \\
\text { variées. } \\
\text { - Vérifier sa } \\
\text { démarche. }\end{array}$ & $\begin{array}{l}\text { - Résoudre des } \\
\text { problèmes à la } \\
\text { manière } \\
\text { d'experts. } \\
\text {-Écrire, } \\
\text { discuter et } \\
\text { étayer sa } \\
\text { pensée. }\end{array}$ & $\begin{array}{l}\text { - Utiliser les } \\
\text { outils selon la } \\
\text { discipline. } \\
\text { - Représenter la } \\
\text { démarche } \\
\text { (scientifique). }\end{array}$ & $\begin{array}{l}\text { - Passer de la } \\
\text { lecture du texte à } \\
\text { la représentation } \\
\text { visuelle pour } \\
\text { illustrer sa } \\
\text { compréhension } \\
\text { (littératie visuelle } \\
\text { et numérique). }\end{array}$ & $\begin{array}{l}\text { - Utiliser les } \\
\text { propriétés du } \\
\text { langage } \\
\text { disciplinaire. } \\
\text { - Utiliser la } \\
\text { notation } \\
\text { symbolique de } \\
\text { la discipline. }\end{array}$ & $\begin{array}{l}\text { - S'approprier } \\
\text { différents genres de } \\
\text { communication. } \\
\text { - S'approprier les } \\
\text { normes d'édition. }\end{array}$ \\
\hline
\end{tabular}




\begin{tabular}{|c|c|c|c|c|c|c|}
\hline 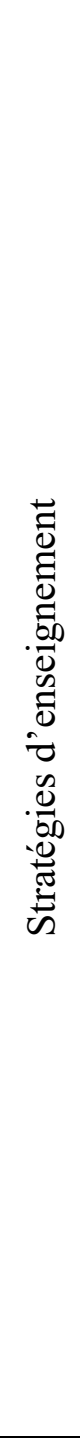 & $\begin{array}{l}\text { - Enrichir le } \\
\text { vocabulaire. } \\
\text { - Modeler une } \\
\text { séquence en } \\
\text { fonction d'un } \\
\text { but ou d'une } \\
\text { hypothèse. } \\
\text { - Enseigner } \\
\text { explicitement } \\
\text { l'organisation } \\
\text { en tableau, en } \\
\text { schéma ou en } \\
\text { organisateur } \\
\text { graphique. }\end{array}$ & $\begin{array}{l}\text { - Identifier, } \\
\text { recueillir, } \\
\text { classer les } \\
\text { informations } \\
\text { en fonction } \\
\text { des } \\
\text { phénomènes à } \\
\text { expliquer. } \\
\text { - Formuler des } \\
\text { hypothèses. } \\
\text { - Vérifier les } \\
\text { hypothèses. } \\
\text { - Écrire une } \\
\text { conclusion } \\
\text { qui tienne } \\
\text { compte de la } \\
\text { démarche } \\
\text { empruntée. }\end{array}$ & $\begin{array}{l}\text { - Décoder le } \\
\text { vocabulaire } \\
\text { (préfixe, } \\
\text { suffixe, mots de } \\
\text { même famille). } \\
\text { - Activer les } \\
\text { connaissances } \\
\text { antérieures. } \\
\text { - Se questionner } \\
\text { avant, pendant } \\
\text { et après la } \\
\text { lecture. } \\
\text { - Enseigner } \\
\text { explicitement } \\
\text { l'inférence, des } \\
\text { modes de } \\
\text { raisonnement. } \\
\text { - Enseigner } \\
\text { explicitement } \\
\text { les genres } \\
\text { textuels, leurs } \\
\text { caractéristiques } \\
\text { et leurs utilités. } \\
\text { - Enseigner } \\
\text { explicitement } \\
\text { les procédés } \\
\text { discursifs } \\
\text { propres à la } \\
\text { discipline } \\
\text { (science). }\end{array}$ & $\begin{array}{l}\text { - Clarifier le } \\
\text { vocabulaire } \\
\text { propre aux } \\
\text { concepts } \\
\text { enseignés. } \\
\text { - Analyser la } \\
\text { structure du texte } \\
\text { pour y trouver } \\
\text { des informations } \\
\text { (ex. rapport de } \\
\text { laboratoire, texte } \\
\text { informatif, } \\
\text { manuel de classe, } \\
\text { etc.). } \\
\text { - Justifier selon la } \\
\text { méthode } \\
\text { disciplinaire } \\
\text { (scientifique). }\end{array}$ & $\begin{array}{l}\text { Enseigner } \\
\text { explicitement } \\
\text { les procédés } \\
\text { discursifs } \\
\text { propres à la } \\
\text { discipline. }\end{array}$ & $\begin{array}{l}\text { - S'approprier le } \\
\text { vocabulaire } \\
\text { spécifique pour } \\
\text { rapporter, } \\
\text { reformuler ou } \\
\text { justifier les } \\
\text { observations, les } \\
\text { connaissances, les } \\
\text { démarches. } \\
\text { - S'approprier des } \\
\text { méthodes pour } \\
\text { communiquer ses } \\
\text { résultats soit à l'oral } \\
\text { ou à l'écrit. }\end{array}$ \\
\hline
\end{tabular}


Prenant appui sur le contenu du tableau 2, notre intention est de montrer quelles sont les considérations à prendre en compte par l'enseignant dans sa planification, notamment au regard de son rôle de médiateur et d'accompagnateur de l'apprentissage qui lui incombe (Roegiers, 2016). Le cas de la rédaction d'un rapport de laboratoire constitue un exemple concret d'un type d'écrit propre à la science et à la technologie (voir Tableau 3). Ce genre textuel commande une présentation typique de la démarche scientifique, dont les étapes doivent être mises en évidence tout en respectant un ordre établi. La formulation de la démarche étayée en étapes requiert l'utilisation de verbes à l'infinitif et la formulation d'hypothèses qui repose sur les connaissances préalables de l'élève quant aux phénomènes impliqués dans l'expérimentation. Ces connaissances sont formulées selon des phrases courtes. Les résultats doivent être représentés visuellement sous forme de tableaux, graphiques ou schémas. La discussion doit référer aux connaissances préalables et par le recours à des critères théoriques qui ont été précédemment expliqués en classe. Enfin, la conclusion doit être formulée selon les conventions scientifiques de façon à valider ou non l'hypothèse de départ. Si la rédaction du rapport de laboratoire constitue en soi une situation complexe d'apprentissage et d'évaluation des compétences à lire et à écrire, il est nécessaire que l'enseignement favorise l'appropriation de certaines habiletés en littératie générale chez les élèves au préalable.

Comme suggéré par Bulgren et al. (2007), les élèves s'approprieront davantage les modalités, stratégies ou moyens s'ils leur sont enseignés et s'ils bénéficient de tâches suffisamment fréquentes et diversifiées pour qu'ils apprennent. Dans cet exemple en particulier, un enseignement du vocabulaire spécifique est requis de même que des stratégies d'inférence sont à introduire au sein de la planification. Enseigner aux élèves à s'interroger permet de prédire, d'inférer et d'interroger de nouveaux contenus. Cet enseignement de stratégies de haut niveau en lecture aide les élèves à maîtriser la compréhension de textes propres à la discipline, de documents numériques et de livres liés à la science, et améliore la performance scientifique globale. De plus, l'enseignement des inférences permet d'intégrer la lecture, l'écriture et l'oral comme modalités d'expression de la pensée critique. L'utilisation des connecteurs de relation aide à l'articulation de leur pensée, de leur discours oral et écrit. La planification qui appartient à l'enseignant constitue alors la pierre d'assise du développement des compétences en littératie générale et de leur transférabilité en contexte de littératie disciplinaire, notamment en science et technologie (Kaldenberg et al., 2015). 
Tableau 3

Barème de présentation du rapport de laboratoire en science et technologie au secondaire

\begin{tabular}{|c|c|c|}
\hline \multicolumn{3}{|c|}{ «Les régions du tableau périodiques » } \\
\hline Étape & Action & Exemple \\
\hline 1. But(s) & $\begin{array}{l}\text { Énoncer par un verbe à l'infinitif le ou les } \\
\text { objectifs à atteindre. }\end{array}$ & Déterminer la nature d'un élément. \\
\hline 2. Hypothèse(s) & $\begin{array}{l}\text { Formuler clairement vos prédictions à partir du } \\
\text { but. } \\
\text { (Se baser sur les connaissances scientifiques } \\
\text { vues en classe). }\end{array}$ & $\begin{array}{l}\text { Je crois que mon élément est un non-métal parce qu'il a un aspect } \\
\text { terne (sans éclat) et parce qu'il est malléable. }\end{array}$ \\
\hline 3. Cadre théorique & $\begin{array}{l}\text { Identifier les connaissances théoriques qui } \\
\text { vous serviront à atteindre votre but. }\end{array}$ & $\begin{array}{l}\text { Il y a trois natures possibles dans l'identification d'un élément. } \\
\text { L'élément peut être un métal, un non-métal ou un métalloïde. } \\
\text { Propriétés caractéristiques : } \\
\text { a) métaux: } \\
\text { - Ils ont un éclat métallique. } \\
\text { - Ils sont de bons conducteurs (thermiques et électriques). } \\
\text { - Ils sont malléables et ductiles. } \\
\text { - Plusieurs d'entre eux réagissent avec les acides. } \\
\text { b) non-métaux: } \\
\text { - Ils ont un aspect terne, sans éclat. } \\
\text { - Ils sont de mauvais conducteurs (thermiques et électriques). } \\
\text { - À } 20 \text { Celsius, on peut les voir dans les } 3 \text { phases (solide, } \\
\text { liquide ou gaz). } \\
\text { b) métalloïdes: } \\
\text { - Ils ont un mélange de propriétés (métalliques et non } \\
\text { métalliques). } \\
\text { - Ils sont de mauvais conducteurs (thermiques et électriques). } \\
\text { - Ressemble aux non-métaux, mais sont de mauvais } \\
\text { conducteurs. }\end{array}$ \\
\hline 4. Protocole & $\begin{array}{l}\text { Énumérer en colonne tous les } \\
\text { instruments avec leur capacité. }\end{array}$ & $\begin{array}{l}\text { - Un élément inconnu } \\
\text { - Appareil à conductibilité électrique }\end{array}$ \\
\hline
\end{tabular}




\begin{tabular}{|c|c|c|c|c|c|c|c|}
\hline & & \multicolumn{3}{|c|}{$\begin{array}{l}\text { Énumérer les produits avec } \\
\text { leurs particularités. }\end{array}$} & \multicolumn{3}{|c|}{ Acide chlorhydrique à10g/l } \\
\hline 4. Protocole & $\begin{array}{l}\text { Manipulation } \\
\mathrm{s}\end{array}$ & \multicolumn{2}{|c|}{$\begin{array}{l}\text { Énumérer et numéroter les } \\
\text { étapes de l'expérimentation. } \\
\text { - Utiliser des verbes à l'infinitif } \\
\text { pour débuter la phrase. } \\
\text { - Faire des phrases courtes. }\end{array}$} & \multicolumn{4}{|c|}{$\begin{array}{l}\text { 1. Vérifier la conductibilité électrique de l'élément avec l'aide de } \\
\text { l'appareil prévu à cette fin (s'assurer que les deux électrodes de } \\
\text { l'appareil soient en contact avec l'élément). } \\
\text { 2. Vérifier l'éclat de l'élément avec la lumière. } \\
\text { 3. Plier l'élément pour vérifier sa malléabilité. } \\
\text { 4. Déposer quelques gouttes d'acide chlorhydrique sur l'élément et } \\
\text { vérifier s'il y a de l'effervescence ou non. } \\
\text { 5. Noter mes résultats dans un tableau. }\end{array}$} \\
\hline \multirow[t]{3}{*}{ 5. Résultats } & \multicolumn{7}{|c|}{ Présenter les données sous forme de tableau de ce que vous avez observé (qualitatif) ou mesuré (quantitatif). } \\
\hline & & $\begin{array}{l}\text { Élémen } \\
\mathrm{t} \\
\text { inconnu }\end{array}$ & $\begin{array}{l}\text { Conductibilit } \\
\text { é électrique }\end{array}$ & Éclat & Malléabilité & $\begin{array}{l}\text { Réaction } \\
\text { à l'acide }\end{array}$ & Nature \\
\hline & & 2 & OUI & \begin{tabular}{|l} 
Terne \\
sans \\
éclat \\
\end{tabular} & NON & NON & Métalloïde \\
\hline 6. Analyse & c) Discussion & \multicolumn{3}{|c|}{$\begin{array}{l}\text { Répondre au but de } \\
\text { l'expérimentation en utilisant vos } \\
\text { résultats et en les comparant avec la } \\
\text { théorie. }\end{array}$} & \multicolumn{3}{|c|}{$\begin{array}{l}\text { Mon élément inconnu est un métallö̈de. Comme on peut le } \\
\text { voir dans le cadre théorique, mon élément ressemble aux non- } \\
\text { métaux. Il a un aspect terne (sans éclat). Il est non malléable. } \\
\text { Il ne fait pas d'effervescence (pas de réaction avec l'acide } \\
\text { chlorhydrique). Cependant, il possède une caractéristique des } \\
\text { métaux. Mon élément est conducteur d'électricité. Étant donné } \\
\text { que mon élément possède des propriétés métalliques et non } \\
\text { métalliques, je peux affirmer qu'il fait partie des métalloüdes. }\end{array}$} \\
\hline \multirow{2}{*}{$\begin{array}{l}\text { 7. Conclusion } \\
\text { ( } 2 \text { étapes) }\end{array}$} & \multicolumn{4}{|c|}{$\begin{array}{l}\text { a) Énoncer clairement le résultat final de } \\
\text { l'expérience en répondant à votre but. }\end{array}$} & \multicolumn{3}{|c|}{ Mon élément inconnu est un métalloïde. } \\
\hline & \multicolumn{4}{|c|}{ b) Mentionner si l'hypothèse est confirmée ou non. } & \multicolumn{3}{|c|}{$\begin{array}{l}\text { Mon hypothèse est fausse, car si mon élément était un non- } \\
\text { métal, il ne serait pas conducteur d'électricité. }\end{array}$} \\
\hline
\end{tabular}


L'exemple décrit au tableau 3 vise essentiellement à rappeler toute l'importance du rôle de l'enseignant qui consiste notamment à intégrer les interventions reconnues par la recherche. Dans notre premier article portant sur la recension des recherches antérieures en littératie (Granger et Moreau, soumis), nous avons relevé bon nombre d'interventions en enseignement encore peu observées dans le milieu scolaire. Plusieurs chercheurs (Graham et Harris, 2009; Rouhani, Moulavie Nafchi et Mehdi Ziaee, 2016) soulignent que les élèves en difficulté peinent à prendre des notes, à surligner et à composer des phrases explicatives, entre autres. Ils ne savent pas comment catégoriser, structurer les informations en tenant compte des idées principales et secondaires. Lorsqu'ils doivent composer, la plupart de ces élèves cherchent à copier des passages directement du texte plutôt qu'à composer leurs propres phrases. Ces constats mènent les chercheurs à conclure que les interventions visant à soutenir l'appropriation de stratégies d'écriture et d'habiletés cognitives font encore peu partie des interventions en classe. En ce sens, ils recommandent d'offrir du soutien et de l'accompagnement aux enseignants pour leur permettre d'intégrer dans leur pratique ces interventions reconnues par la recherche.

\section{Des obstacles à la mise en cuvre d'interventions en littératie}

Soucieux de produire une synthèse des connaissances qui soit utile aux chercheurs, mais aussi aux enseignants, nous avons d'abord cherché du côté de la littératie générale puis du côté de la littératie disciplinaire. Cependant, ni la recension d'Hillman (2014), ni celle de Flaggella-Luby et al. (2012) ne traite de la façon dont l'enseignant doit s'y prendre pour structurer les séquences d'apprentissage pour ses élèves. Toujours dans la perspective de guider la planification, nous avons consulté le Programme de formation de l'école québécoise (MEQ, 2003) et la progression des apprentissages publiée par le Ministère de l'Éducation, du Loisir et du Sport (MELS) en 2011 afin de trouver des repères. Or, les informations contenues dans ces deux documents relèvent davantage de grandes orientations que d'interventions concrètes à intégrer dans l'enseignement disciplinaire pour favoriser le développement de compétences de tous les élèves à lire, à écrire et à développer leur pensée critique.

Ce constat nous amène à rejoindre les conclusions des auteurs cités précédemment (Flaggella-Luby et al., 2012; Flynn, Zheng et Swanson, 2012; Graham et Harris, 2009; Hillman, 2014; Kaldenberg et al., 2014; McKenna, Shin et Ciullo, 2015) pour souligner l'urgence de documenter les interventions ou pratiques effectives en salle de classe. Nous pensons qu'il ne suffit pas de relever les bonnes pratiques pour préconiser le changement en enseignement, il convient d'offrir des modèles diversifiés, des exemples concrets de planifications et d'interventions dont les enseignants pourront s'inspirer pour planifier des séquences d'enseignement-apprentissage qui intègrent des interventions reconnues en littératie.

De plus, la réflexion que nous avons amorcée, nous a mené à vouloir mieux comprendre la planification au regard de l'évaluation des compétences en tant que moyen pour réguler deux types de finalités soit les compétences développées en cours d'apprentissage et celles attendues comme finalité certificative. Ces deux intentions régulent nécessairement la planification de l'enseignant qui doit planifier ses interventions en fonction de nombreux paramètres (contenu essentiel- savoirs, stratégies d'apprentissage -, savoir-faire, intention pédagogique en fonction des compétences visées, etc.). Or, actuellement au Québec, les programmes de formation ne rendent pas la tâche facile à un enseignant qui souhaiterait construire des séquences d'enseignement qui s'appuient sur les interventions pédagogiques recommandées dans les recherches. Ainsi, nous faisons le constat d'un besoin chez les enseignants de pouvoir s'appuyer sur des documents ministériels plus explicites qui leur permettraient de mieux planifier et de mieux formaliser les interventions attendues en contexte scolaire. 
À cet égard, les articles recensés dans nos deux articles présentent des résultats qui s'étendent sur plus de 30 ans de recherche en éducation. Il ne s'agit plus de se questionner sur leur efficacité, mais d'une part d'en vérifier leur effet par des recherches dans le milieu et, d'autre part, de les intégrer réellement dans les programmes de formation. Ce pas de plus permettrait aux futurs enseignants de s'en inspirer et de les mettre en œuvre dès leur arrivée dans le système scolaire, nonobstant l'ordre ou la discipline d'enseignement. En ce sens, une grande responsabilité incombe aux décideurs. Une certaine cohésion entre la formation en enseignement et les connaissances issues de la recherche contribuerait certainement à soutenir le développement des compétences en lecture et en écriture chez tous les élèves et à optimiser leur potentiel.

\section{Conclusion}

Dans cet article, l'analyse de recensions antérieures et de recherches sur l'enseignement de la littératie disciplinaire en contexte d'inclusion a permis de préciser l'incidence de ces savoirs sur la planification de l'enseignement. Les articles analysés nous ont permis de définir la littératie scolaire au regard de trois termes soit la littératie de base intermédiaire que constituent la littératie générale et la littératie disciplinaire (haut niveau de compétences). Outre l'apparente convergence qui existe entre certains auteurs qui préconisent l'importance d'une littératie plutôt qu'une autre, l'analyse de ces écrits scientifiques nous a permis d'y voir une complémentarité essentielle pour l'enseignement et l'apprentissage. Ainsi, Flaggella-Luby et al. (2012) montrent bien la nécessité de favoriser des interventions en littératie générale qui s'adressent à tous les élèves et plus spécifiquement à ceux ayant des difficultés d'apprentissage. Ces auteurs soulignent à quel point ces interventions sont de nature transdisciplinaire et permettent de tisser des liens dans différentes disciplines sur la façon d'aborder les textes, de les comprendre et d'en rendre compte. D'autre part, la recension d'Hillman (2014) nous sensibilise à l'importance de la posture disciplinaire de l'enseignant et d'une bonne connaissance des méthodes d'enseignement en littératie propre à son champ de compétence, soit le comment faire. Ces auteurs font ressortir le rôle que doit jouer l'enseignant au moment de planifier ses séquences d'enseignement-apprentissage. Cette recension nous a permis de mieux comprendre les implications d'interventions ciblées en littératie pour habiliter l'ensemble des élèves lorsqu'il s'agit de lire, d'écrire et de développer sa pensée critique en enseignement disciplinaire. Il serait pertinent de réfléchir aux moyens à déployer pour soutenir l'application de ces connaissances dans le milieu scolaire notamment en contexte de transition du primaire au secondaire, espace où l'on constate davantage de désengagement de la part des élèves et une recrudescence de l'échec scolaire. Ces recherches doivent cibler tant les élèves typiques que ceux ayant des difficultés.

\section{Références}

Bostic, J., \& Matney, G. (2013). Overcoming a common storm: Designing professional development for teachers implementing the Common Core. Ohio Journal of School Mathematics, 67, 12-19.

Bourassa, M., Bélair, L., \& Chevalier, J. (2007). Les outils de la recherche participative. Éducation et Francophonie, 35(2), 1-11.

Bulgren, J. A., Lenz, B. K., Schumaker, J. B., Deshler, D. D., \& Marquis, J. G. (2002). The use and effectiveness of a comparison routine in diverse secondary content classrooms. Journal of Educational Psychology, 94(2), 356-371.

Bulgren, J. A., Deshler, D. D., \& Lenz, B. K. (2007). Engaging adolescents with LD in higher order thinking about history concepts using integrated content enhancement routines. Journal of Learning Disabilities, 40(2), 121-133. 
Edmonds, M. S., Vaughn, S., Wexler, J., Reutebuch, C., Cable, A., Klingler Tackett, K., \& al. (2009). A synthesis of reading interventions and effects on reading comprehension outcomes for older struggling readers. Review of Educational Research, 79(1), 262-300.

Flagella-Luby, M. N., \& Deshler, D. D. (2008). Reading comprehension in adolescents with LD: What we know; what we need to learn. Learning Disabilities Research \& Practice, 23(2), 70-78.

Flaggella-Luby, M.N., Sampson Graner, P., Deshler. D. D., \& Valentino Drew, S. (2012). Building a house on sand: Why disciplinary literacy is not sufficient to replace general strategies for adolescent learners who struggle. Topics in Language Disorders, 32(1), 6984.

Flynn, L. J., Zheng, X., \& Swanson, H. (2012). Instructing struggling older readers: A selective meta-analysis of intervention research. Learning Disabilities Research and Practice, 27, 21-32.

Fortin, M.-F. \& Gagnon, J. (2016). Fondements et étapes du processus de recherche. Méthodes quantitatives et qualitatives. Montréal : Chenelière éducation.

Gee, J.P. (2012). Social linguistics and literacies: Ideology in Discourses. New York, NY: Routledge.

Graham, S. \& Harris, K.R. (2009). Almost 30 Years of Writing Research: Making Sense of It All with The Wrath of Khan. Learning Disabilies Rresearch and Practice, 24(2), 58-68.

Graham, S., \& Perin, D. (2007). A meta-analysis of writing instruction for adolescent students. Journal of Educational Psychology, 99(3), 445-476.

Granger, N. \& Moreau, A.C. (2018). Que disent les recensions antérieures de recherches sur les enseignements favorisant les compétences en littératie de la fin du primaire et du secondaire? Language and literacy.

Gresalfi, M., Martin, T., Hand, V., \& Greeno, J. (2009). Constructing competence: An analysis of student participation in the activity systems of mathematics classrooms. Educational Studies in Mathematics, 70, 49-70.

Harris, K. R., \& Graham, S. (1999). Programmatic intervention research: Illustrations from the evolu- tion of self-regulated strategy development. Learning Disability Quarterly, 22(4), 251-262.

Hillman, A.M. (2014). A literature review on disciplinary literacy: How do secondary teacher s apprentice students mathematical literacy? Journal of Adolescent and Adult Literacy, 57(5), 397-406.

Jackson, G. B. (1980). Methods for integrative reviews. Review of Educational Research, 50(3), 438-460.

Jonnaert, P. (2010). Approche par situations - Matrice du traitement compétent d'une situation. Cahiers de la CUDC, 5, numéro thématique.

Kaldenberg, E. R., Watt, S.J., \& Therrien, W.J. (2015). Reading Instruction in Science for Students With Learning Disabilities: A Meta-Analysis. Learning Disability Quarterly, 38(3), 160-173.

Lacelle, N., Lafontaine, L., Moreau, A.C., \& Laroui, R. (2016). Réseau québécois sur la littératie. Repéré à http://www.ctreq.qc.ca/realisation/reseau-quebecois-sur-la-litteratie/

Lee, J., Grigg, W., \& Donahue, P. (2007). The nation's re-port card: Reading 2007 (NCES No. 2007-496). Washington, DC: National Center for Education Statistics, Institute of Education Sciences, U.S. Department of Education.

Lenoir, Y. (2009). L'intervention éducative, un construit théorique pour analyser les pratiques d'enseignement. Nouveaux cahiers de la recherche en éducation, 12(1), 9-29. 
McKenna, J.W., Shin, M., \& Ciullo, S. (2015). Evaluating reading and mathematics instruction for students with learning disabilities: A synthesis of observation research. Learning disability quarterly, 38(4), 195-2017.

Messier, G. (2014). Proposition d'un réseau conceptuel initial qui précise et illustre la nature, la structure ainsi que la dynamique des concepts apparentés au terme méthode en pédagogie. (Thèse de doctorat). Montréal : Université du Québec à Montréal.

Ministère de l'Éducation du Québec (MEQ) (2003). Programme de formation à l'école québécoise. Enseignement secondaire: premier cycle. Québec : Gouvernement du Québec.

Ministère de l'Éducation, du Loisir et du Sport (MELS) (2011). La progression des apprentissages au secondaire. Québec : Gouvernement du Québec. Repéré à http://www1.education.gouv.qc.ca/progressionSecondaire/pdf/progrApprSec_ST_PFG_fr2011-11-24.pdf

Moreau, A.C., Hébert, M., Lépine, M., \& Ruel, J. (2013). Le concept de littératie en francophonie : que disent les définitions? Revue Consortium national de recherche sur l'intégration sociale (CNRIS), 4(2), 14-18.

National Center for Education Statistics (2009). The nation's report card: Reading 2009. Washington, DC: National Center for Education Statistics, Institute of Education Sciences, U.S. Department of Education.

Nesbit, J. C., \& Adesope, O. O. (2006). Learning with concept and knowledge maps: A metaanalysis. Review of Educational Research, 76(3), 413-448.

Organisation de coopération et de développement économique (OCDE) (2000). La littératie à l'ère de l'information. Rapport final de l'Enquête internationale sur la littératie des adultes.

Passmore, T. (2007). Polya's legacy: Fully forgotten or getting a new perspective in theory and practice? Australian Senior Mathematics Journal, 21(2), 44-53. SEp?

Perle, M., Grigg, W., \& Donahue, P. (2005). The nation's report card: Reading 2005 (NCES2006-451). Washington, DC: U.S. Department of Education, National Center for Education Statistics.

Roegiers, X. (2010). La Pédagogie de l'Intégration. Des Systèmes d'Éducation et de Formation au Coeur de nos Sociétés. Bruxelles: De Boeck.

Roegiers, X. (2016). Un cadre conceptuel pour l'évaluation des compétences. Réflexion en cours $n^{\circ} 4$ sur Les principaux enjeux actuels en matière de curriculum et d'apprentissage. Bureau international d'éducation de l'UNESCO (BIE).

Rouhani, Y., Moulavi Nafchi, A., \& Mehdi Ziaee, S. (2016). Applying Different Interventions to Teach Writing to Students with Disabilities: A Review Study. Theory and Practice in Language Studies, 6(4), 733-741.

Sfard, A. (2007). When the rules of discourse change, but nobody tells you: Making sense of mathematics learning from a cognitive standpoint. The Journal of the Learning Sciences, $16(4), 565-613$.

Shanahan, T., \& Shanahan, C. (2008). Teaching disciplinary literacy to adolescents: Rethinking content area literacy. Harvard Educational Review, 78(1), 40-59.

Shanahan, T., \& Shanahan, C. R. (2012). What is disciplinary literacy and why does it matter? Topics in Language Disorders, 32(1), 7-18.

Schumaker, J. B., \& Deshler, D. D. (2009). Adolescents with learning disabilities as writers: Are we selling them short? Learning Disabilities Research \& Practice, 24(2), 81-92.

Scruggs, T. E., \& Mastropieri, M. A. (2000). The effectiveness of mnemonic instruction for students with learning and behavior problems: An update and research synthesis. Journal 
of Behavioral Education, 10(2-3), 163-173.

Tardif, J. (2006). L'Évaluation des Compétences. Documenter le Parcours de Développement. Montréal: Chenelière Éducation.

Thévenaz-Christen, T. (2011). La littératie, un concept ? Forum Lecture, ch. 1.

Trabasso, T., \& Bouchard, E. (2002). Teaching readers how to comprehend text strategically. Dans C. C. Block \& M. Pressley (dir.), Comprehension instruction: Research-based best practices (pp. 176-200). New York: Guilford Press.

Wiggins, G. P. (1993). Assessing Student Performance. San Francisco: Jossey-Bass Publishers.

Wilkerson-Jerde, M.H., \& Wilensky, U.J. (2011). How do mathematicians learn math? Resources and acts for con- structing and understanding math. Educational Studies in Mathematics, 78(1), 21-43.

Zimmerman, B. J., \& Schunk, D. H. (2001). Self-regulated learning and academic achievement: theoretical perspectives (2nd ed.). Mahwah, NJ: Erlbaum.

Zorica, I.M., Cindric, M., \& Destovic, F. (2012). Traditional and contemporary approaches to teaching mathematics. Technics Technologies Education Management, 7(2), 882-888.

Biographie des auteures

Nancy Granger, Ph. D. est professeur adjointe à l'Université de Sherbrooke où elle est coresponsable du Microprogramme de $2^{e}$ cycle en conseillance pédagogique au département de Gestion de l'éducation et de la formation. Elle est chercheuse au sein de l'Équipe de recherche en littératie et inclusion (ÉRLI) où elle a réalisé un postdoctorat. Ses recherches portent sur la formation continue et l'accompagnement des enseignants, l'inclusion scolaire des élèves ayant des besoins particuliers, la littératie ainsi que sur les pratiques d'enseignement et d'apprentissage. Des recherches-actions ont également été réalisées sur le thème de l'organisation des services notamment au regard de la fonction d'enseignant-ressource au secondaire et sur le dispositif de coenseignement. Des articles scientifiques et chapitres de livres ont été publiés sur ces différents thèmes.

André C. Moreau, Ph. D. est professeur agrégé à l'Université du Québec en Outaouais. Ses recherches portent sur la littératie et l'inclusion des élèves ayant des besoins particuliers et sur les pratiques d'enseignement et d'apprentissage. Il est cotitulaire de la Chaire interdisciplinaire de recherche en littératie et inclusion - CISSS de l'Outaouais ainsi que chercheur d'Équipe de recherche en littératie et inclusion (ÉRLI). Des recherches-actions ont également été réalisées sur les thèmes de transitions scolaires et de communautés d'apprentissage professionnelles. Il a publié plusieurs articles scientifiques, livres et rapports de recherche sur ces thèmes. 